PROCEEDINGS OF THE

AMERICAN MATHEMATICAL SOCIETY

Volume 134, Number 8, Pages 2239-2250

S 0002-9939(06)08559-5

Article electronically published on March 14, 2006

\title{
ON BIACCESSIBLE POINTS OF THE MANDELBROT SET
}

\author{
SAEED ZAKERI
}

(Communicated by Linda Keen)

\begin{abstract}
This paper provides a description for the quadratic polynomials on the boundary of the Mandelbrot set $\mathcal{M}$ which are typical in the sense of harmonic measure. In particular, it is shown that a typical point on the boundary of $\mathcal{M}$ has a unique parameter ray landing on it. Applications of this result in the study of embedded $\operatorname{arcs}$ in $\mathcal{M}$ and the lamination associated with $\mathcal{M}$ are given.
\end{abstract}

\section{INTRODUCTION}

Consider the complex quadratic family $\left\{f_{c}: z \mapsto z^{2}+c\right\}_{c \in \mathbb{C}}$ and its connectedness locus $\mathcal{M}$, commonly known as the "Mandelbrot set." This paper will give a short proof of the following

Theorem 1. There exists a Borel set $X \subset \partial \mathcal{M}$ of full harmonic measure such that for every $c \in X$ :

(i) the Julia set $J_{c}=J\left(f_{c}\right)$ is locally connected and full;

(ii) the post-critical orbit $\left\{c, f_{c}(c), f_{c}^{\circ 2}(c), \ldots\right\}$ is evenly distributed with respect to the harmonic measure on $J_{c}$ (in particular it is dense in $J_{c}$ );

(iii) $\mathcal{M}$ is locally connected at $c$, only one parameter ray lands at $c$, the impression of this ray is trivial, and no other parameter ray contains $c$ in its impression. Moreover, the dynamic ray of the same angle lands at the critical value $c \in J_{c}$.

The statement can be recovered from the work of Bruin and Schleicher $[\mathrm{BSc}$ in which they use symbolic dynamics to study various combinatorial and topological aspects of the quadratic family. Modulo the uniqueness of rays in (iii), it also follows from the work of Smirnov S1] and Graczyk and Świątek GS. Instead of using their results, here we present a very elementary argument based on Yoccoz's local connectivity theorem and the topology of trees in Julia sets.

Recall that $c \in \partial \mathcal{M}$ is biaccessible if two or more parameter rays land at $c$. Thus, we have the following corollary of Theorem 1 .

Corollary 2. The set of all biaccessible points in the Mandelbrot set has harmonic measure zero.

Received by the editors February 5, 2004 and, in revised form, January 24, 2005.

2000 Mathematics Subject Classification. Primary 37F10, 37F20, 37F35, 35F45.

(C)2006 American Mathematical Society Reverts to public domain 28 years from publication 
The analogue of this result has been known for all connected quadratic Julia sets, with the exception of the Julia set of the Chebyshev map $f_{-2}: z \mapsto z^{2}-2$, which is a straight line segment (see [S2], [Z1], [Zd]).

As another corollary, in $\$$ we show that

Theorem 3. Every embedded arc in the Mandelbrot set has harmonic measure zero.

In particular, the real slice $\mathcal{M} \cap \mathbb{R}=[-2,1 / 4]$ of the Mandelbrot set has harmonic measure zero. In [Z2], this result came as a byproduct of a completely different method.

Let $\Lambda_{\mathcal{M}} \subset \mathbb{D}$ be the lamination associated with $\mathcal{M}$ (see $₫ 5$ for the definition and compare Figure 3).

Theorem 4. The area of $\Lambda_{\mathcal{M}}$ is zero, and its Hausdorff dimension is two.

The proof, presented in $\$ 5$, will depend on Theorem 1 and the fact that the set of angles of the parameter rays which land on the real slice of $\mathcal{M}$ has Hausdorff dimension one [Z2].

Let us point out that the area statement in Theorem 4 bears resemblance to Thurston's observation that geodesic laminations on hyperbolic surfaces of finite area have measure zero (see for example [Th, Chapter 8]). On the other hand, the dimension statement in Theorem 4 is in sharp contrast with the theorem of Birman and Series according to which such geodesic laminations always have Hausdorff dimension one [BS]. It may be worthwhile to further study the metric structure of laminations that arise in complex dynamics (such as $\Lambda_{\mathcal{M}}$ and those associated with Julia sets) and develop a theory parallel to geodesic laminations on hyperbolic surfaces.

\section{Preliminaries}

First we briefly recall some facts about the conformal geometry of the complement of a planar continuum; see $[\underline{\mathrm{A}}, \underline{\mathrm{Mc}}$, $\underline{\mathrm{M}}$ 1 or $[\mathrm{P}$ for details. Let $K \subset \mathbb{C}$ be a non-degenerate continuum. Assume further that $K$ is full in the sense that its complement $\mathbb{C} \backslash K$ is connected. By the Riemann mapping theorem, there exists a unique conformal isomorphism $\psi_{K}: \mathbb{C} \backslash \overline{\mathbb{D}} \cong \mathbb{C} \backslash K$ which satisfies $\lim _{z \rightarrow \infty} \psi_{K}(z) / z>0$. By the external ray at angle $t \in \mathbb{T}=\mathbb{R} / \mathbb{Z}$ is meant the analytic curve $R_{K}(t)=\psi_{K}\left(\left\{r e^{2 \pi i t}: r>1\right\}\right)$. We say that $R_{K}(t)$ lands at $p \in \partial K$ if $\lim _{r \rightarrow 1} \psi_{K}\left(r e^{2 \pi i t}\right)=p$. According to Carathéodory, $\psi_{K}$ extends continuously to the boundary circle $\partial \mathbb{D}$ if and only if $K$ is locally connected, in which case all external rays land [M1, Theorem 17.14]. Whether or not $K$ is locally connected, a theorem of Beurling asserts that $R_{K}(t)$ lands for all $t$ outside a set of logarithmic capacity zero [P] Theorem 9.19].

The (prime-end) impression $I_{K}(t)$ of the ray $R_{K}(t)$ is the set of all $z \in \partial K$ for which there is a sequence $\left\{w_{n}\right\}$ in $\mathbb{C} \backslash \overline{\mathbb{D}}$ such that $w_{n} \rightarrow e^{2 \pi i t}$ and $\psi_{K}\left(w_{n}\right) \rightarrow z$. It is not hard to show that $I_{K}(t)$ is a non-empty sub-continuum of $\partial K$, and $\bigcup_{t \in \mathbb{T}} I_{K}(t)=$ $\partial K$. Here is another easy consequence of the definition of impression that we will need in the proof of Theorem 4 in $\$ 5$

$$
\limsup _{n \rightarrow \infty} I_{K}\left(t_{n}\right) \subset I_{K}(t) \quad \text { whenever } \quad t_{n} \rightarrow t .
$$


When $I_{K}(t)$ reduces to a point, we say that it is a trivial impression. This happens precisely when $\psi_{K}$ has a continuous extension to $e^{2 \pi i t}$. A ray with trivial impression necessarily lands, but a landing ray may well have a non-trivial impression.

A point $p \in \partial K$ is accessible if it is the landing point of an external ray, and is biaccessible if at least two external rays land on it. It can be shown that $p$ is biaccessible if and only if $K \backslash\{p\}$ is disconnected. Given a subset $E \subset K$, define

$$
\psi_{K}^{-1}(E)=\left\{t \in \mathbb{T}: R_{K}(t) \text { lands at a point of } E\right\} .
$$

The continuum $K$ decomposes into the disjoint union $K=K^{0} \cup K^{1} \cup K^{2} \cup \cdots \cup K^{\infty}$, where

$$
K^{n}=\left\{p \in K: \psi_{K}^{-1}(p) \text { has exactly } n \text { elements }\right\}, \quad 0 \leq n \leq \infty .
$$

It is easy to verify that each $K^{n}$ is a Borel measurable subset of $K$, and $K^{0}$ contains the interior of $K$ (possibly empty). Note that in terms of this decomposition, a point is accessible if and only if it belongs to $K \backslash K^{0}$, and is biaccessible if and only if it belongs to $K \backslash\left(K^{0} \cup K^{1}\right)$.

The normalized 1-dimensional Lebesgue measure on $\partial \mathbb{D} \cong \mathbb{T}$ pushes forward by the Riemann map $\psi_{K}$ to a Borel measure supported on $\partial K$ called the harmonic measure. In other words, the harmonic measure of a Borel subset $E \subset K$ is given by the length of the set $\psi_{K}^{-1}(E)$ defined in (2.2). By the theorem of Beurling mentioned above, the set $K^{0}$ has harmonic measure zero. The same is true for $K^{3} \cup \cdots \cup K^{\infty}$, since this union is easily seen to be a countable set [P. Corollary 2.19] and single points in $K$ have harmonic measure zero by a theorem of F. and M. Riesz [M1, Theorem A.3]. It follows that $K=K^{1} \cup K^{2}$ up to a set of harmonic measure zero.

We now give a brief account of a few basic results in the dynamics of the complex quadratic family; further details can be found in [DH1, [DH2, [Mc, M2] and [M3]. Let $c \in \mathbb{C}$. The filled Julia set of $f_{c}: z \mapsto z^{2}+c$, denoted by $K_{c}$, is the set of all points in the plane with bounded forward orbit under $f_{c}$. The topological boundary $J_{c}=\partial K_{c}$ is called the Julia set of $f_{c}$. Both sets are non-empty, compact, and totally invariant, with $K_{c}$ being full. An external ray for $K_{c}$ is called a dynamic ray. By a cycle of $f_{c}$ is meant a periodic orbit $z \mapsto f_{c}(z) \mapsto \cdots \mapsto f_{c}^{\circ p}(z)=z$. The quantity $\lambda=\left(f_{c}^{\circ p}\right)^{\prime}(z)$ is called the multiplier of this cycle. The cycle is attracting, repelling, or indifferent according to whether $|\lambda|<1,|\lambda|>1$, or $|\lambda|=1$. A parameter $c$ is Misiurewicz if the forward orbit under $f_{c}$ of the critical point 0 is finite but not periodic.

The Mandelbrot set $\mathcal{M}$ consists of all parameters $c \in \mathbb{C}$ for which the (filled) Julia set of $f_{c}$ is connected. Equivalently, $c \in \mathcal{M}$ if and only if $0 \in K_{c}$. It can be shown that $\mathcal{M}$ is a full continuum. We will use the term parameter ray for an external ray of $\mathcal{M}$. A parameter $c \in \mathcal{M}$ is called hyperbolic if the corresponding quadratic $f_{c}$ has a necessarily unique attracting cycle in the plane. Hyperbolic parameters appear as connected components of the interior of $\mathcal{M}$. The main hyperbolic component $H_{0}$ consists of all $c \in \mathcal{M}$ for which $f_{c}$ has an attracting fixed point. It is the prominently visible cardioid in any picture of $\mathcal{M}$ and is defined by the inequality $|1-\sqrt{1-4 c}|<1$ (compare Figure 3 left). The period of a hyperbolic component $H$ is the length of the unique attracting cycle of $f_{c}$ for any $c \in H$. There is a canonical conformal isomorphism $\lambda_{H}: H \stackrel{\cong}{\longrightarrow}$ which assigns to each $c \in H$ the multiplier of its attracting cycle. The map $\lambda_{H}$ extends to a homeomorphism $\bar{H} \cong \overline{\mathbb{D}}$. The root of $H$ is by definition the point $\lambda_{H}^{-1}(1) \in \partial H$. We call $H$ a satellite component if its root is on the boundary of another hyperbolic component. There are exactly 
two parameter rays of angles $\theta_{-}<\theta_{+}$landing at the root of $H$ which are rational numbers of the form $n /\left(2^{p}-1\right)$, where $p$ is the period of $H$. (When $H=H_{0}$, the two angles $\theta_{-}=0$ and $\theta_{+}=1$ coincide, and only one ray lands at the root $c=1 / 4$.) It follows that these two angles have binary expansions of the form

$$
\theta_{-}=0 . \overline{\theta_{0}} \quad \text { and } \quad \theta_{+}=0 . \overline{\theta_{1}},
$$

where $\theta_{0}$ and $\theta_{1}$ are binary words of length $p$, and the bars indicate infinite repetition. Conversely, if $t$ is a rational of odd denominator, the parameter ray $R_{\mathcal{M}}(t)$ lands at the root of a unique hyperbolic component.

For every hyperbolic component $H$, denote by $\iota_{H}: \mathcal{M} \hookrightarrow \mathcal{M}$ the DouadyHubbard tuning map (see [DH1] or [M3]). Then $\iota_{H}$ is a topological embedding which maps $H_{0}$ onto $H$, respects roots of hyperbolic components, and maps $\partial \mathcal{M}$ into $\partial \mathcal{M}$. The image $M_{H}=\iota_{H}(\mathcal{M})$ is a subset of $\mathcal{M}$ homeomorphic to $\mathcal{M}$, with $\partial H \subset \partial M_{H} \subset \partial \mathcal{M}$. We have $M_{H_{0}}=\mathcal{M}$, but when $H \neq H_{0}, M_{H}$ is a proper subset of $\mathcal{M}$ often described as the small copy of $\mathcal{M}$ growing from $H$.

The tuning operation also acts on parameter rays. Let $\theta_{-}=0 . \overline{\theta_{0}}<\theta_{+}=0 . \overline{\theta_{1}}$ be the angles of the two parameter rays landing at the root of a period- $p$ hyperbolic component $H \neq H_{0}$ as in (2.3). For any angle $t \in \mathbb{T}$ with the binary expansion 0. $t_{1} t_{2} t_{3} \cdots$, define the tuned angle

$$
A_{H}(t)=0 . \theta_{t_{1}} \theta_{t_{2}} \theta_{t_{3}} \cdots
$$

by concatenating blocks of words of length $p>1$. Note that under this tuning algorithm on angles, a dyadic rational has two distinct images since it has two different binary representations. The image $A_{H}(\mathbb{T})$ is a self-similar Cantor set and as such has length zero. In fact, let $\Lambda_{i}: \mathbb{T} \rightarrow \mathbb{T}(i=0,1)$ be the map defined by $\Lambda_{i}\left(0 . t_{1} t_{2} t_{3} \cdots\right)=0 . \theta_{i} t_{1} t_{2} t_{3} \cdots$. Then $\Lambda_{i}$ is an affine contraction by a factor $2^{-p}$ and the image $A_{H}(\mathbb{T})$ is precisely the invariant set generated by the pair $\Lambda_{0}, \Lambda_{1}$ [Ma]. It can be shown that if $R_{\mathcal{M}}(t)$ lands at $c \in M$, then the parameter ray $R_{\mathcal{M}}\left(A_{H}(t)\right)$ lands at the tuned image $\iota_{H}(c) \in M_{H}$ [D1]. Furthermore, with countably many possible exceptions, these are the only parameter rays that land on the small copy $M_{H}$. As a result,

$$
\operatorname{length}\left(\psi_{\mathcal{M}}^{-1}\left(M_{H}\right)\right)=\operatorname{length}\left(A_{H}(\mathbb{T})\right)=0 \quad \text { if } H \neq H_{0} .
$$

Finally, a quadratic $f_{c}$ with $c \in \mathcal{M}$ is simply renormalizable of period $p>1$ if there are topological disks $U$ and $V$ containing 0 such that (i) $U$ is compactly contained in $V$; (ii) $f^{\circ p}: U \rightarrow V$ is a degree 2 branched covering; (iii) $0 \in E$, where $E$ is the set of points in $U$ whose forward orbits under $f^{\circ p}$ are contained in $U$; and (iv) the images $E, f(E), \ldots, f^{\circ p-1}(E)$ do not disconnect one another. Simply renormalizable quadratics correspond to parameters in small copies of $\mathcal{M}$. More precisely, if $f_{c}$ is simply renormalizable of period $p>1$, then $c \in M_{H}$ for some period- $p$ hyperbolic component $H$. Conversely, if $H$ is a period- $p$ hyperbolic component with $p>1$, every $c \in M_{H}$ (with the exception of the root of $H$ in the satellite case) gives a quadratic $f_{c}$ which is simply renormalizable of period $p$ [DH1].

\section{Proof of Theorem 1}

The proof of Theorem 1 begins as follows. The set $\mathcal{M}_{\text {ren }} \subset \mathcal{M}$ of parameters $c$ for which the quadratic $f_{c}$ is simply renormalizable is contained in the countable 
union $\bigcup_{H \neq H_{0}} M_{H}$ of all small copies. Thus,

$$
\psi_{\mathcal{M}}^{-1}\left(\mathcal{M}_{\text {ren }}\right) \subset \bigcup_{H \neq H_{0}} \psi_{\mathcal{M}}^{-1}\left(M_{H}\right) .
$$

Since by (2.4) each $\psi_{\mathcal{M}}^{-1}\left(M_{H}\right)$ has length zero, it follows that $\mathcal{M}_{\text {ren }}$ has harmonic measure zero.

For each irreducible fraction $0<p / q<1$ there is a period- $q$ hyperbolic component $H_{p / q}$ whose root is $\lambda_{H_{0}}^{-1}\left(e^{2 \pi i p / q}\right) \in \partial H_{0}$ (see Figure 3 left for the case $p / q=1 / 3$ ). The two parameter rays landing at this root have angles of the form $\theta_{-}=n /\left(2^{q}-1\right)$ and $\theta_{+}=(n+1) /\left(2^{q}-1\right)$. For $t$ in the open interval $I_{p / q}=\left(\theta_{-}, \theta_{+}\right) \subset \mathbb{T}$, the parameter ray $R_{\mathcal{M}}(t)$ does not land on $\partial H_{0}$. It follows that $\psi_{\mathcal{M}}^{-1}\left(\partial H_{0}\right) \subset$ $\mathbb{T} \backslash \bigcup_{0<p / q<1} I_{p / q}$. Since the intervals $I_{p / q}$ are disjoint, we obtain

$$
\operatorname{length}\left(\psi_{\mathcal{M}}^{-1}\left(\partial H_{0}\right)\right) \leq 1-\sum_{0<p / q<1} \frac{1}{2^{q}-1} .
$$

An easy computation gives $\sum_{0<p / q<1} 1 /\left(2^{q}-1\right)=1$ (compare [D1] or [GM]) and we conclude that $\partial H_{0}$ has harmonic measure zero.

Let $\mathcal{M}_{\text {ind }} \subset \mathcal{M}$ be the set of parameters $c$ for which $f_{c}$ has an indifferent cycle. These are precisely the parameters which lie on the boundary of hyperbolic components of $\mathcal{M}$, so

$$
\mathcal{M}_{\text {ind }}=\partial H_{0} \cup \bigcup_{H \neq H_{0}} \partial H \subset \partial H_{0} \cup \bigcup_{H \neq H_{0}} M_{H} .
$$

Since each set on the right has harmonic measure zero, it follows that the harmonic measure of $\mathcal{M}_{\text {ind }}$ is zero.

Now consider the Borel set $Y=\partial \mathcal{M} \backslash\left(\mathcal{M}_{\text {ren }} \cup \mathcal{M}_{\text {ind }}\right)$ consisting of all $c$ for which the quadratic $f_{c}$ is non-renormalizable and without indifferent cycles. By the above discussion, $Y$ has full harmonic measure. According to Yoccoz, for every $c \in Y$ the Julia set $J_{c}$ is a locally connected full continuum. Moreover, $\mathcal{M}$ is locally connected at $c$, only finitely many parameter rays land at $c$, and the dynamic rays of the same angles land at the critical value $c$ of $f_{c}$ (see $[\mathrm{H}],[\mathrm{M} 2,[\mathrm{R}]$ ). This statement can be improved by showing that the parameter rays landing at $c$ have trivial impressions, and no other ray can contain $c$ in its impression. In the case $c$ is a Misiurewicz parameter, this is shown by Tan Lei, building upon the earlier work of Douady and Hubbard [T1. In the case $c$ is non-Misiurewicz, the sharper statement follows from Tan Lei's result and the proof of Yoccoz's theorem. In fact, Yoccoz constructs a nested sequence of para-puzzles $\left\{\Pi_{n}\right\}$ with the following properties:

- $\Pi_{n}$ is a closed topological disk containing $c$;

- $\Pi_{n} \cap \mathcal{M}$ is connected;

- $\partial \Pi_{n} \cap \mathcal{M}$ consists of finitely many Misiurewicz parameters; and

- $\bigcap \Pi_{n}=\{c\}$

(compare $[\underline{\mathbb{R}})$. Let $c$ belong to some impression $I_{\mathcal{M}}(t)$, and assume $\partial \Pi_{n} \cap I_{\mathcal{M}}(t) \neq \emptyset$ for some $n$. Since this intersection is contained in $\partial \Pi_{n} \cap \mathcal{M}$, it could only be a finite set $\left\{c_{1}, \ldots, c_{k}\right\}$ of Misiurewicz parameters. Tan Lei's result would then show that $I_{\mathcal{M}}(t)=\left\{c_{j}\right\}$ for some $j$. This would imply $c=c_{j}$, which would contradict the assumption of $c$ being non-Misiurewicz. Thus, $\partial \Pi_{n} \cap I_{\mathcal{M}}(t)=\emptyset$ and so $I_{\mathcal{M}}(t) \subset \Pi_{n}$ for all $n$. It follows that $I_{\mathcal{M}}(t)=\{c\}$ and in particular $R_{\mathcal{M}}(t)$ lands at $c$. 
Let $c \in Y$ so that $J_{c}$ is locally connected and full. The normalized Riemann map $\psi_{J_{c}}: \mathbb{C} \backslash \overline{\mathbb{D}} \rightarrow \mathbb{C} \backslash J_{c}$ induces a continuous map $\partial \mathbb{D} \cong \mathbb{T} \rightarrow J_{c}$ which conjugates the action of the doubling map $t \mapsto 2 t$ to $f_{c}$ :

$$
\psi_{J_{c}}(2 t)=f_{c}\left(\psi_{J_{c}}(t)\right) \text {. }
$$

If the parameter ray $R_{\mathcal{M}}(t)$ lands at $c$, the dynamic ray $R_{J_{c}}(t)$ lands at the critical value $c \in J_{c}$, and it follows by induction that $R_{J_{c}}\left(2^{k} t\right)$ lands at $f_{c}^{\circ k}(c)$ for all $k \geq 0$. Since the harmonic measure on $J_{c}$ is the push-forward of Lebesgue measure on $\mathbb{T}$ under $\psi_{J_{c}}$, it follows that the post-critical orbit $\left\{c, f_{c}(c), f_{c}^{\circ 2}(c), \ldots\right\}$ is evenly distributed on $J_{c}$ whenever the orbit $t \mapsto 2 t \mapsto 4 t \mapsto \cdots$ is evenly distributed on $\mathbb{T}$. (Recall that a sequence $\left\{x_{n}\right\}$ in a topological space $X$ is evenly distributed with respect to a probability measure $\mu$ if

$$
\lim _{N \rightarrow \infty} \frac{1}{N} \sum_{n=1}^{N} \phi\left(x_{n}\right)=\int_{X} \phi(x) d \mu(x)
$$

for every bounded continuous function $\phi: X \rightarrow \mathbb{R}$.) Since the doubling map is ergodic with respect to Lebesgue measure on the circle, the Ergodic Theorem shows that there is a full length Borel set $B \subset \mathbb{T}$ consisting of angles $t$ whose orbits $t \mapsto 2 t \mapsto 4 t \mapsto \cdots$ are evenly distributed on $\mathbb{T}$. The intersection $\psi_{\mathcal{M}}^{-1}(Y) \cap B$ has full length, so the Borel set $X \subset Y$ which satisfies $\psi_{\mathcal{M}}^{-1}(X)=\psi_{\mathcal{M}}^{-1}(Y) \cap B$ has full harmonic measure. Moreover, the preceding remark shows that for each $c \in X$, the post-critical orbit $\left\{c, f_{c}(c), f_{c}^{\circ 2}(c), \ldots\right\}$ is evenly distributed on $J_{c}$, and in particular is dense in $J_{c}$.

So far we have verified all the properties of $X$ in Theorem 1 except the uniqueness of rays in (iii). This will be a corollary of the lemma below, which may be of independent interest.

Lemma 5. Let $J_{c}$ be locally connected and full. Suppose two or more dynamic rays land at $c$. Then there exists a forward invariant finite topological tree $T \subset J_{c}$ which contains the post-critical orbit $\left\{c, f_{c}(c), f_{c}^{\circ 2}(c), \ldots\right\}$.

It follows from this lemma that every $c \in X$ is the landing point of a unique parameter ray, for otherwise we could find a finite topological tree $T$ in $J_{c}$ containing the post-critical orbit and use the density of this orbit to conclude that the Julia set $J_{c}=T$ is a finite tree. The only quadratic with this property is the Chebyshev map $f_{-2}: z \mapsto z^{2}-2$ which is post-critically finite (see for example M4, Lemma B.4] or [Z1]). This contradiction completes the proof of Theorem [1.

Proof of Lemma 5. Since $J_{c}$ is locally connected and full, each pair $z, w$ of distinct points in $J_{c}$ can be joined by a unique embedded arc $[z, w]=[w, z]$ in $J_{c}$ homeomorphic to the interval $[0,1] \subset \mathbb{R}$. Let $\beta$ be the fixed point of $f_{c}$ at which the dynamic ray at angle 0 lands, and let $\alpha$ be the other fixed point. The following facts can be easily verified (see [Z1] for details and compare Figure 1):

$$
\begin{aligned}
& \text { If } z \in J_{c} \text { is biaccessible, then } f_{c}^{\circ k}(z) \in[-\beta, \beta] \text { for some } k \geq 0 \text {; } \\
& 0 \in[-\beta, \beta] \text { and } \alpha \in[-\beta, 0] \text {; } \\
& f_{c}([\alpha,-\alpha])=[c, \alpha] \text { and } f_{c}([-\beta, \beta])=[c, \alpha] \cup[\alpha,-\alpha] \cup[-\alpha, \beta]=[c, \beta] \text {; } \\
& \text { if } z \in[-\alpha, \beta] \text {, then } f_{c}(z) \in[\alpha, z] \text {; and } \\
& \text { for every } z \in J_{c}, f_{c}([z, \alpha]) \subset\left[f_{c}(z), \alpha\right] \cup[\alpha, c] \text {. }
\end{aligned}
$$




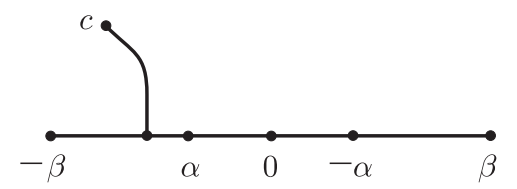

Figure 1. Some embedded arcs in the Julia set $J_{c}$.

Let $c_{k}=f_{c}^{\circ k}(c)$. The assumption that two rays land at $c$ implies by (3.1) that $c_{m} \in[-\beta, \beta]$ for some $m \geq 0$. We claim that there exists $n \geq m$ such that $c_{n} \in[c, \alpha]$. Otherwise, by (3.3), $c_{n} \in[-\alpha, \beta]$ for all $n>m$. It follows from (3.4) that $[-\alpha, \beta] \supset\left[c_{n+1}, \beta\right] \supset\left[c_{n}, \beta\right]$ for all $n>m$. The limit of the monotone sequence $\left\{c_{n}\right\}_{n>m}$ will then be a fixed point of $f_{c}$ in $[-\alpha, \beta]$. Since $\beta$ is the only fixed point of $f_{c}$ in $[-\alpha, \beta]$, this implies $c_{n}=\beta$ for all $n>m$. Since $c$ has two rays landing on it and the forward orbit of $c$ never hits the critical point 0 , every $c_{n}$ and hence $\beta$ must be the landing point of two rays. This is a contradiction since $\beta$ is the landing point of the unique ray at angle 0 .

So choose $n \geq m$ such that $c_{n} \in[c, \alpha]$, or equivalently $\left[c_{n}, \alpha\right] \subset[c, \alpha]$. The union $T=\bigcup_{k=0}^{n-1}\left[c_{k}, \alpha\right]$ is then a forward invariant finite topological tree. In fact, by (3.5), $f_{c}\left(\left[c_{k}, \alpha\right]\right) \subset\left[c_{k+1}, \alpha\right] \cup[\alpha, c] \subset T$ for every $0 \leq k \leq n-1$. Since $c \in T$, the orbit of $c$ is entirely contained in $T$.

\section{EMBedDed ARCS in $\mathcal{M}$}

In this section we prove Theorem 3 using Theorem 1 and the fact that the boundary of every interior component of $\mathcal{M}$ has harmonic measure zero (Lemma 7 below). The argument is based on the following topological lemma.

Lemma 6. Let $K \subset \mathbb{C}$ be a full non-degenerate continuum and let $\gamma \subset K$ be an embedded arc with endpoints $z, w$. Assume there are angles $t, s \in \mathbb{T}$ such that $I_{K}(t)=\{z\}$ and $I_{K}(s)=\{w\}$. Then every point of $\gamma \backslash\{z, w\}$ belongs either to the closure of an interior component of $K$, or to the impression of at least two external rays of $K$.

Note that the assumption on the endpoints of $\gamma$ is necessary. For example, the "comb"

$$
K=[-1,1] \cup[0, i] \cup \bigcup_{n=1}^{\infty}\left[ \pm \frac{1}{n}, \pm \frac{1}{n}+i\right]
$$

has no interior and the point $p=i / 2$ on the embedded arc $\gamma=[0, i]$ belongs to the impression of the unique ray which lands at $i$ (see Figure 22). Note also that the lemma is a generalization of the fact that every point of a "dendrite" (i.e., a locally connected full non-degenerate continuum without interior) is either an end or biaccessible (compare [Z1, Lemma 3]).

Proof. Assume $p \in \gamma \backslash\{z, w\}$ does not belong to the closure of any interior component of $K$. The union $R_{K}(t) \cup \gamma \cup R_{K}(s)$ separates the plane into two connected components $C^{+}$and $C^{-}$. Take a sequence of open topological disks $D_{n}$ around $p$ such that diam $D_{n} \rightarrow 0$ and $D_{n} \backslash\left(R_{K}(t) \cup \gamma \cup R_{K}(s)\right)$ has two connected components $D_{n}^{+}$and $D_{n}^{-}$, labelled so that $D_{n}^{+} \subset C^{+}$and $D_{n}^{-} \subset C^{-}$for all $n$. For each $n$, both topological disks $D_{n}^{ \pm}$intersect $\mathbb{C} \backslash K$, for otherwise one of them would be 


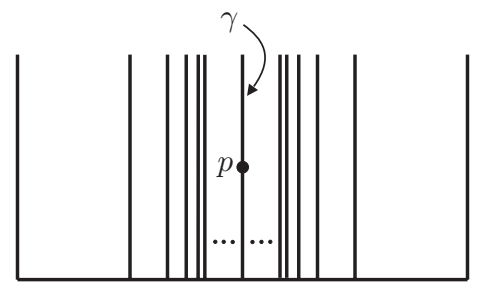

FIGURE 2.

contained in the interior of $K$ and $p$ would be in the closure of an interior component. Choose sequences $z_{n}^{ \pm} \in D_{n}^{ \pm} \backslash K$ and write $z_{n}^{ \pm}=\psi_{K}\left(r_{n} \exp \left(2 \pi i t_{n}^{ \pm}\right)\right)$. After passing to subsequences if necessary, we can assume that $t_{n}^{ \pm} \rightarrow t^{ \pm}$. It then follows from the definition of impression that $p \in I_{K}\left(t^{+}\right) \cap I_{K}\left(t^{-}\right)$.

Since $R_{K}\left(t_{n}^{ \pm}\right) \subset C^{ \pm}$, we have $R_{K}\left(t^{ \pm}\right) \subset \overline{C^{ \pm}}$. If $t^{+}=t^{-}$, it follows that $R_{K}\left(t^{+}\right)=R_{K}\left(t^{-}\right) \subset \overline{C^{+}} \cap \overline{C^{-}}$, so $t^{+}=t^{-}=t$ or $t^{+}=t^{-}=s$. But then $p \in I_{K}\left(t^{+}\right) \cap I_{K}\left(t^{-}\right) \subset I_{K}(t) \cup I_{K}(s)$, which implies $p=z$ or $p=w$. This contradicts our assumption. Thus $t^{+} \neq t^{-}$.

Lemma 7. The union of the boundaries of the interior components of $\mathcal{M}$ has harmonic measure zero.

Proof. We show that this union is contained in the harmonic measure zero set $E=\partial H_{0} \cup \bigcup_{H \neq H_{0}} M_{H}$ (see $\oint 3$ ). Let $W$ be an interior component of $\mathcal{M}$. If $W$ is a hyperbolic component, then $\partial W \subset \partial H_{0} \cup \bigcup_{H \neq H_{0}} \partial H \subset E$. Otherwise, $W$ is a "queer" component. In this case, for every $c \in W$ the quadratic $f_{c}$ has no indifferent cycle and the Julia set $J_{c}$ admits non-trivial deformations, hence has positive area [Mc, Theorem 4.9]. A theorem of Lyubich and Shishikura then shows that $f_{c}$ is (infinitely) renormalizable (see $[\mathrm{L}$ or $[\mathrm{Mc}$, Theorem 8.8]). It follows that $W$ is contained in a small copy $M_{H}$ for some $H \neq H_{0}$. So, again, $\partial W \subset M_{H} \subset E$.

Proof of Theorem 3. Let $\gamma \subset \mathcal{M}$ be an embedded arc and assume by way of contradiction that $\gamma$ has positive harmonic measure. Consider the subset $\eta \subset \gamma$ consisting of the parameters which do not belong to the closure of any interior component of $\mathcal{M}$. By Lemma 7 the harmonic measure of the difference $\gamma \backslash \eta$ is zero, so $\eta$ has positive harmonic measure. Let $X \subset \partial \mathcal{M}$ be the set given by Theorem 1 . Since $X$ has full harmonic measure, the intersection $\eta \cap X$ has positive harmonic measure. Choose three distinct parameters $c, c^{\prime}, c^{\prime \prime}$ in $\eta \cap X$, labelled so that $c^{\prime}$ is between $c$ and $c^{\prime \prime}$ on $\gamma$. By Theorem 1(iii), each of these parameters forms the (trivial) impression of a unique external ray. But Lemma 6 applied to the segment of $\gamma$ from $c$ to $c^{\prime \prime}$ shows that $c^{\prime}$ must belong to the impression of at least two rays. This is a contradiction, and the proof is complete.

\section{The lamination associated with $\mathcal{M}$}

We begin with some definitions. A (geodesic) lamination in $\mathbb{D}$ is a relatively closed set $\Lambda \subset \mathbb{D}$ decomposed into a disjoint union of complete Poincaré geodesics. A lamination $\Lambda$ has a local product structure: Every point of $\Lambda$ has a neighborhood $U$ homeomorphic to the unit square $(0,1) \times(0,1)$ such that $\Lambda \cap U$ corresponds to $(0,1) \times \tau$ for some relatively closed set $\tau \subset(0,1)$, and such that the geodesic 


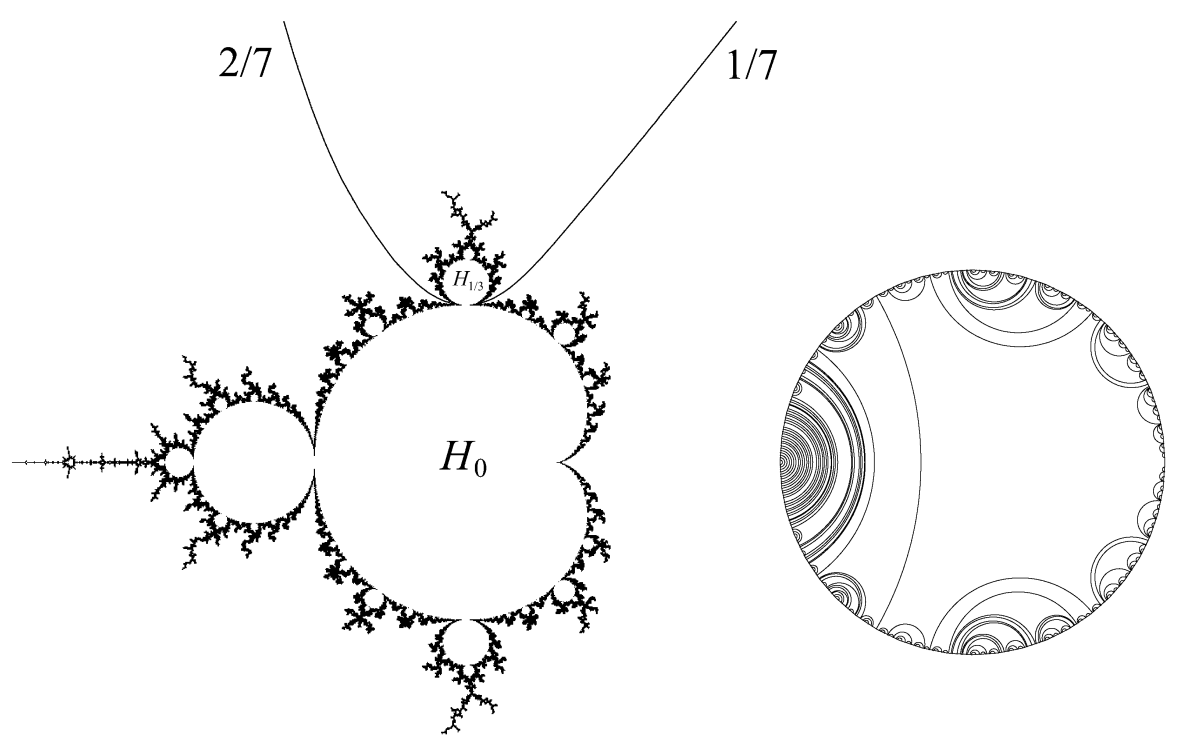

Figure 3. Left: The Mandelbrot set $\mathcal{M}$ with its main hyperbolic component $H_{0}$ and the satellite component $H_{1 / 3}$ at whose root the parameter rays at angles $1 / 7$ and $2 / 7$ land. The small copy $M_{H_{1 / 3}}$ is a proper subset of the part of $\mathcal{M}$ cut off by these rays. Right: The lamination $\Lambda_{\mathcal{M}}$ associated with the Mandelbrot set.

segments in $\Lambda \cap U$ correspond to the horizontal segments $(0,1) \times\{$ point $\}$ (see for example [ $\mathrm{B}$ ). We call $U$ a product neighborhood. $\Lambda$ is said to be a product lamination if it can be covered by a single product neighborhood.

An equivalence relation $\sim$ on $\mathbb{T}$ is closed if its graph $\{(t, s) \in \mathbb{T} \times \mathbb{T}: t \sim s\}$ is a closed subset of $\mathbb{T} \times \mathbb{T}$, and is unlinked if any two distinct equivalence classes are contained in a pair of disjoint intervals. Suppose $\sim$ is closed and unlinked. Identify $\mathbb{T}$ with the unit circle $\partial \mathbb{D}$, and form the convex hull (in the Poincaré metric of $\mathbb{D}$ ) of each equivalence class of $\sim$. Take the union of the topological boundaries of all these convex hulls and define $\Lambda$ as the intersection of this union with the unit disk $\mathbb{D}$. It is not hard to check that $\Lambda$ is a geodesic lamination in $\mathbb{D}$. We refer to it as the lamination of $\sim$.

In the case of the Mandelbrot set, define the equivalence relation $\sim_{\mathbb{Q}}$ on $\mathbb{Q} / \mathbb{Z}$ by declaring $t \sim_{\mathbb{Q}} s$ if and only if the parameter rays $R_{\mathcal{M}}(t)$ and $R_{\mathcal{M}}(s)$ land at the same point. Extend $\sim_{\mathbb{Q}}$ to a relation $\sim$ on the circle $\mathbb{T}$ by taking the closure of the graph of $\sim_{\mathbb{Q}}$, that is, declare $t \sim s$ if and only if there are sequences of rational angles $t_{n} \rightarrow t$ and $s_{n} \rightarrow s$ such that $t_{n} \sim_{\mathbb{Q}} s_{n}$ for all $n$. It turns out that $\sim$ is an equivalence relation, is closed and unlinked, and for $t, s \in \mathbb{Q} / \mathbb{Z}, t \sim s$ if and only if $t \sim_{\mathbb{Q}} s$ [D2]. The lamination $\Lambda_{\mathcal{M}}$ of this equivalence relation is called the lamination associated with $\mathcal{M}$ (see Figure 3 right). Douady and Hubbard have used $\Lambda_{\mathcal{M}}$ to construct a combinatorial model of $\mathcal{M}$ known as the "abstract Mandelbrot set" (see [DH1] or [D2]).

The following lemma will be used in the proof of Theorem 4

Lemma 8. Let $\Lambda \subset \mathbb{D}$ be a product lamination. If the closure $\bar{\Lambda}$ meets $\partial \mathbb{D}$ along a set of length zero, then the area of $\Lambda$ must be zero. 
Proof. Since $\Lambda$ is a product lamination, it is homeomorphic to $(0,1) \times \tau$ for some relatively closed set $\tau \subset(0,1)$. It suffices to prove the lemma when $\tau$ is compact; the general case will then follow by writing $\Lambda=\bigcup \Lambda_{n}$, where $\Lambda_{n}$ is the product sub-lamination of $\Lambda$ which is homeomorphic to $(0,1) \times\left(\tau \cap\left[\frac{1}{n}, 1-\frac{1}{n}\right]\right)$. Assuming $\tau$ is compact, the closure $\bar{\Lambda}$ is homeomorphic to $[0,1] \times \tau$, and $\bar{\Lambda} \cap \partial \mathbb{D}$ consists of two disjoint compact parts $\tau_{0} \cong\{0\} \times \tau$ and $\tau_{1} \cong\{1\} \times \tau$, both having length zero.

Let $J_{0}$ and $J_{1}$ be disjoint open intervals in $\partial \mathbb{D}$ containing $\tau_{0}$ and $\tau_{1}$, respectively. The holonomy $h: \tau_{0} \stackrel{\cong}{\longrightarrow} \tau_{1}$ given by following the geodesics in $\Lambda$ can be extended to a homeomorphism $h: J_{0} \stackrel{\cong}{\longrightarrow} J_{1}$ (for example by interpolating linearly on each component of $\left.J_{0} \backslash \tau_{0}\right)$. Since both $\tau_{0}$ and $\tau_{1}$ have zero length, given $\varepsilon>0$ there is an open neighborhood $V \subset J_{0}$ of $\tau_{0}$ such that length $(V)<\varepsilon$ and length $(h(V))<\varepsilon$. Decompose $V$ into the disjoint union of open intervals $\left\{I_{n}\right\}$, so that $h(V)$ is the disjoint union of open intervals $\left\{h\left(I_{n}\right)\right\}$. Let $U_{n} \subset \overline{\mathbb{D}}$ be the convex hull of $I_{n} \cup h\left(I_{n}\right)$ in the Poincare metric on the disk. The Euclidean area of the quadrilateral $U_{n}$ is easily estimated by

$$
\operatorname{area}\left(U_{n}\right) \leq C\left(\text { length }\left(I_{n}\right)+\text { length }\left(h\left(I_{n}\right)\right)\right),
$$

where $C>0$ is an absolute constant. Since $\Lambda \subset \bigcup U_{n}$, it follows that

$$
\begin{aligned}
\operatorname{area}(\Lambda) & \leq \sum \operatorname{area}\left(U_{n}\right) \leq C \sum\left(\operatorname{length}\left(I_{n}\right)+\operatorname{length}\left(h\left(I_{n}\right)\right)\right) \\
& =C(\operatorname{length}(V)+\operatorname{length}(h(V))) \leq 2 C \varepsilon,
\end{aligned}
$$

which shows $\operatorname{area}(\Lambda)=0$.

Proof of Theorem 4. Let $\sim$ be the equivalence relation used in the definition of $\Lambda_{\mathcal{M}}$. If $t \sim s$ and $t \neq s$, there exist sequences of rational angles $t_{n} \rightarrow t$ and $s_{n} \rightarrow s$ such that $t_{n} \sim_{\mathbb{Q}} s_{n}$ for all $n$. Let $z_{n}$ be the common landing point of $R_{\mathcal{M}}\left(t_{n}\right)$ and $R_{\mathcal{M}}\left(s_{n}\right)$. Then, by (2.1), any accumulation point of the sequence $\left\{z_{n}\right\}$ must belong to both impressions $I_{\mathcal{M}}(t)$ and $I_{\mathcal{M}}(s)$; in particular, $I_{\mathcal{M}}(t) \cap I_{\mathcal{M}}(s) \neq \emptyset$. If $X \subset \partial \mathcal{M}$ is the set given by Theorem 1, it follows that every equivalence class of $\sim$ containing more than one element must be disjoint from $\psi_{\mathcal{M}}^{-1}(X)$. This means that the set of endpoints of the geodesics in $\Lambda_{\mathcal{M}}$ is a subset of $\mathbb{T} \backslash \psi_{\mathcal{M}}^{-1}(X)$, which has length zero.

Let $U$ be any product neighborhood for $\Lambda_{\mathcal{M}}$ and let $\Lambda$ be the union of all geodesics in $\Lambda_{\mathcal{M}}$ which intersect $U$. Then $\Lambda$ is a product lamination, and by the preceding paragraph $\bar{\Lambda} \cap \partial \mathbb{D}$ has length zero. By Lemma 8 , area $(\Lambda)=0$. Since $\Lambda \supset \Lambda_{\mathcal{M}} \cap U$, we conclude that area $\left(\Lambda_{\mathcal{M}} \cap U\right)=0$. Since $\Lambda_{\mathcal{M}}$ can be covered by countably many such product neighborhoods $U$, we conclude that area $\left(\Lambda_{\mathcal{M}}\right)=0$.

Now consider the set $\psi_{\mathcal{M}}^{-1}(\mathbb{R})$ of angles $t$ for which the parameter ray $R_{\mathcal{M}}(t)$ lands on the real line. It is shown in $\left[\mathbf{Z 2}\right.$ that $\psi_{\mathcal{M}}^{-1}(\mathbb{R})$ is a nowhere dense set of Hausdorff dimension 1 , and that $t \sim-t$ for every $t \in \psi_{\mathcal{M}}^{-1}(\mathbb{R})$. It easily follows that the union over all $t \in \psi_{\mathcal{M}}^{-1}(\mathbb{R})$ of the Poincaré geodesic in $\mathbb{D}$ joining $t$ to $-t$ has Hausdorff dimension 2. Since this union is contained in $\Lambda_{\mathcal{M}}$, we conclude that the dimension of $\Lambda_{\mathcal{M}}$ must be 2 .

\section{ACKNOWLEDGMENTS}

I am indebted to Jacek Graczyk who brought the results of [GS] to my attention, which prompted the writing of this note. I am also grateful to John Milnor for his useful comments on an earlier draft of the paper. 


\section{REFERENCES}

[A] L. Ahlfors, Conformal invariants, McGraw-Hill, 1973. MR0357743 (50:10211)

[BS] J. Birman and C. Series, Geodesics with bounded intersection number on surfaces are sparsely distributed, Topology, 24 (1985) 217-225. MR.0793185 (87f:57012)

[B] F. Bonahon, Geodesic laminations on surfaces, in "Laminations and foliations in dynamics, geometry and topology," Contemp. Math., 269, Amer. Math. Soc. 2001, 1-37. MR1810534 (2001m:57023)

[BSc] H. Bruin and D. Schleicher, Symbolic dynamics of quadratic polynomials, Mittag-Leffler Institute preprints, 2001/2002, No. 07.

[D1] A. Douady, Algorithms for computing angles in the Mandelbrot set, in "Chaotic Dynamics and Fractals," Academic Press, 1986, 155-168. MR.0858013

[D2] A. Douady, Descriptions of compact sets in $\mathbb{C}$, in "Topological methods in modern mathematics," Publish or Perish, 1993, 429-465. MR.1215973 (94g:58185)

[DH1] A. Douady and J. Hubbard, Etude dynamique de polynommes complexes, Publications Math. d'Orsay 84-02 (1984) (premiere partie) and 85-04 (1985) (deuxieme partie). MR.0762431 (87f:58072a) MR.0812271 (87f:58072b)

[DH2] A. Douady and J. Hubbard, On the dynamics of polynomial-like mappings, Ann. Sci. Ec. Norm. Sup., 18 (1985) 287-343. MR0816367 (87f:58083)

[GM] L. Goldberg and J. Milnor, Fixed points of polynomial maps II, Ann. Sci. Ec. Norm. Sup., 26 (1993) 51-98. MR.1209913 (95d:58107)

[GS] J. Graczyk and G. Swiątek, Harmonic measure and expansion on the boundary of the connectedness locus, Invent. Math., 142 (2000) 605-629. MR.1804163 (2001k:37066)

[H] J. Hubbard, Local connectivity of Julia sets and bifurcation loci, in "Topological Methods in Modern Mathematics," Publish or Perish, 1993, 457-511. MR1215974 (94c:58172)

[L] M. Lyubich, On the Lebesgue measure of the Julia set of a quadratic polynomial, Stony Brook IMS preprint \# 1991/10.

[Ma] A. Manning, Logarithmic capacity and renormalizability for landing on the Mandelbrot set, Bull. London Math. Soc., 28 (1996) 521-526. MR1396155 (97g:58141)

[Mc] C. McMullen, Complex Dynamics and Renormalization, Annals of Math Studies 135, Princeton University Press, 1994. MR 1312365 (96b:58097)

[M1] J. Milnor, Dynamics in one complex variable: Introductory lectures, Vieweg, 1999. MR:1721240(2002i:37057)

[M2] J. Milnor, Local connectivity of Julia sets: expository lectures, in "The Mandelbrot set, Theme and Variations," LMS Lecture Note Series 274, Cambr. U. Press 2000, 67-116. MR 1765085 (2001b:37073)

[M3] J. Milnor, Periodic orbits, external rays and the Mandelbrot set: An expository account, Astérisque, 261 (2000) 277-333. MR:1755445(2002e:37067)

[M4] J. Milnor, Pasting together Julia sets: a worked out example of mating, Experiment. Math., 13 (2004) 55-92. MR 2065568 (2005c:37087)

[P] C. Pommerenke, Boundary behaviour of conformal maps, Springer-Verlag, 1992. MR.1217706 (95b:30008)

[R] P. Røsch, Holomorphic motions and puzzles, in "The Mandelbrot set, Theme and Variations," LMS Lecture Note Series 274, Cambr. U. Press 2000, 117-131. MR1765086 (2001c:37046)

[S1] S. Smirnov, Symbolic dynamics and the Collet-Eckmann condition, Internat. Math. Res. Notices, 7 (2000) 333-351. MR1749741 (2001i:37071)

[S2] S. Smirnov, On support of dynamical laminations and biaccessible points in Julia set, Colloq. Math., 87 (2001) 287-295. MR1814670 (2001m:37092)

[T1] Tan Lei, Voisinages connexes des points de Misiurewicz, Ann. Inst. Fourier Grenoble, 42 (1992) 707-735. MR1196091 (94a:58165)

[T2] Tan Lei, Local properties of the Mandelbrot set at parabolic points, in "The Mandelbrot set, Theme and Variations," LMS Lecture Note Series 274, Cambr. U. Press 2000, 133-160. MR.1765087 (2001g:37065)

[Th] W. Thurston, The geometry and topology of 3-manifolds, Lecture notes, Princeton University, 1976-1979.

[Z1] S. Zakeri, Biaccessibility in quadratic Julia sets, Ergod. Th. \& Dyn. Sys., 20 (2000) 18591883. MR 1804961 (2001k:37068) 
[Z2] S. Zakeri, External rays and the real slice of the Mandelbrot set, Ergod. Th. \& Dyn. Sys., 23 (2003) 637-660. MR.1972243 (2004a:37057)

[Zd] A. Zdunik, On biaccessible points in Julia sets of polynomials, Fund. Math., 163 (2000) 277-286. MR:1758329 (2001f:37058)

Institute for Mathematical Sciences, Stony Brook University, Stony Brook, New YORK 11794

Current address: Department of Mathematics, Queens College of CUNY, Flushing, New York 11367

E-mail address: zakeri@forbin.qc.edu 